\title{
Influence of tool pin and shoulder geometries on microstructure of friction stir processed AA6063/SiC composites
}

\author{
Namrata Gangil ${ }^{1, *}$, Sachin Maheshwari ${ }^{1}$, and Arshad Noor Siddiquee ${ }^{2}$ \\ ${ }^{1}$ Division of Manufacturing Processes and Automation Engineering, Netaji Subhas Institute of Technology, 110078 New Delhi, \\ India \\ ${ }^{2}$ Department of Mechanical Engineering, Jamia Millia Islamia, 110025 New Delhi, India
}

Received: 14 December 2016 / Accepted: 10 November 2017

\begin{abstract}
The present study investigated the combined effect of friction stir processing (FSP) tool pin and shoulder profiles on particle distribution and microstructure of AA6063/SiC composites. Two strategies were used, in first strategy, plain cylindrical, tapered cylindrical, square and triangular tool pin profiles were used with flat shoulder design. In second strategy square and cylindrical pin profiles were used along with clock-wise (CW) and anti clock-wise (ACW) scrolled shoulder design. Single pass processing was performed to fabricate the composites. Microstructure examination of sample processed with various pin profiles and flat shoulder design reveals that tool with square pin profile was most effective. Among other shoulder profiles, ACW scrolled shoulder resulted in pancake shaped stir zone (SZ) and exhibited significant improvement in SZ size, out of which ACW scrolled shoulder with cylindrical pin profile produced uniform particle distribution without any defects.
\end{abstract}

Keywords: Friction stir processing / aluminum alloy / tool pin profile / tool shoulder geometry / particle distribution

\section{Introduction}

Friction Stir Processing (FSP) is based on the principle of Friction Stir Welding (FSW) developed at "The Welding Institute (TWI), UK" in 1991 [1]. In FSP a cylindrical tool is made to plunge, while rotating, in to base metal (BM) and traversed on the workpiece surface in the processing direction as shown in Figure 1. For fabricating surface composites the matrix material is first preplaced with reinforcement particles. The rubbing action of tool shoulder generates frictional heat and softens material under the shoulder which also undergoes severe plastic deformation at high strain rate by the rotating pin and mixes the reinforcement particles in matrix. During FSW/ FSP, material is subjected to a combination of metal working processes e.g. friction, extrusion and forging [1-4]. FSP is evolving as a promising surface modification technology and a surface composite fabrication process mainly because it is a solid-state and environment friendly process by virtue of being free from use of consumables and affluent. One of the major challenges of the process, however, is inhomogeneous distribution of reinforcement particles. A large number of research works has been focused on achieving homogeneous distribution of

\footnotetext{
* e-mail: namrata.gangil@gmail.com
}

particles, elimination of agglomeration of particles, overcoming of tunnel like defects and achieving wide composite zone by utilizing various strategies such as applying multiple number of FSP passes [5,6], change of tool rotation between passes $[7,8]$, process hybridization such as electric current assisted FSP [9] etc. All these, result in loss of time and energy and often loss of substrate properties as well. Multiple passes not only increase the energy input, production time but every pass also, often lowers the material properties especially in case of age-hardenable alloys.

Studies with specific focus on material flow and reinforcement particle distribution have been reported in which effects of pin profile have been considered [7,10,11]. Systematic investigation on effect of shoulder profile, however, remains under reported with few researches that use concave [8] and scrolled shoulder [9,12] for surface composite fabrication without investigating specifically the combined effect of pin and shoulder profiles on response. The researchers have generally performed parametric studies using some tool geometry of their choice. But, the tool geometry including shoulder profile, pin shape and size has a far more significant effect on distribution of reinforcement particles. It is pertinent that shoulder profile is important to materials movement and hence an important factor in obtaining adequate particle distribution. The combined effect of shoulder profiles coupled with pin profile is even more important and critical to particle 


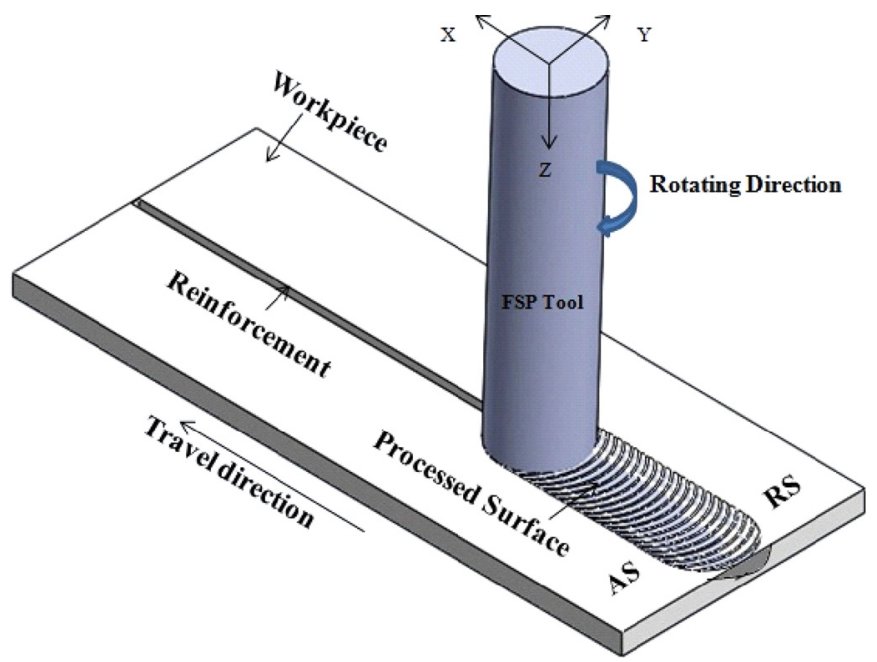

Fig. 1. Schematic diagram of Friction Stir Processing.

distribution. This study is performed with a specific objective to understand the distribution of $\mathrm{SiC}$ particles in AA6063 alloy for which the effect of tool profile (i.e. shoulder profile and pin profile both) has been investigated. It is worthwhile to mention that if a prior decision with regard to correct tool geometry is taken, efficient reinforcement distribution can be obtained without making use of additional number of passes and use of any additional strategic parameter. In this paper shoulder profile and pin profiles both were varied and their effect on particle distribution is analysed. Three profiles e.g. flat and two different scrolls (clockwise and counter-clock wise) shoulder profiles were used. Pin profiles of cylindrical, tapered cylindrical, square and triangular prism shaped pins were selected to investigate the effect of tool profile. Findings of this investigation are important and provide knowledge useful to better tool design and effective tool selection, bring out better distribution in single pass.

\section{Materials and methods}

Experimental samples of $170 \times 50 \times 4.75 \mathrm{~mm}^{3}$ dimensions with slots of $2 \times 2 \mathrm{~mm}^{2}$ size machined in the middle were prepared from 6063-T6 aluminum alloy BM. Slots were filled with $\mathrm{SiC}$ powder $(13 \mu \mathrm{m})$, subsequently covered and compacted using a pinless tool. Single pass FSP runs were carried out on an indigenously developed FSW machine. Initially tools with flat shoulder and four different tool pin profiles (i.e. cylindrical, tapered cylindrical, square prism and triangular prism as shown in Fig. 2) were used. In the second set of four experiments, cylindrical and square prismatic pins coupled with spiral scroll, $\mathrm{CW}$ and ACW (0.5 scroll height and $1 \mathrm{~mm}$ scroll width) shoulder profiles (as shown in Fig. 3) were used.

High-carbon high-chromium ( $\mathrm{HCHCr})$ steel tools having shoulder $24 \mathrm{~mm}$ in diameter, pin $2.5 \mathrm{~mm}$ long and $5 \mathrm{~mm}$ circum-circle diameter were used. Shoulder diameter, size of pin and conventional FSP parameters such as tool rotation speed, traverse speed, target depth and shoulder diameter were selected through comprehensive trials. For

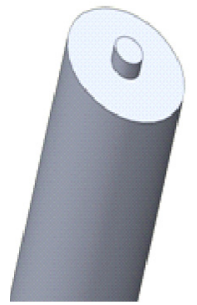

(a)

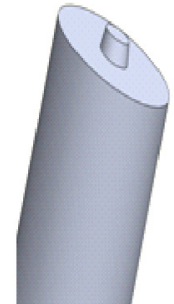

(b)

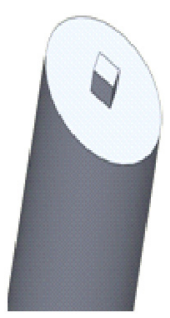

(c)

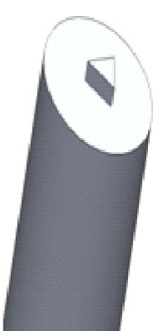

(d)
Fig. 2. Tool Pin Profiles with flat shoulder surface (a) Plain Cylindrical, (b) Tapered Cylindrical, (c) Square, (d) Triangular.

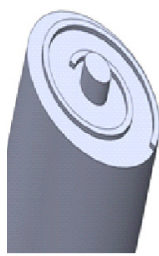

(a)

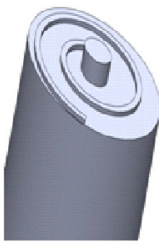

(b)

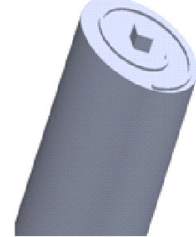

(c)

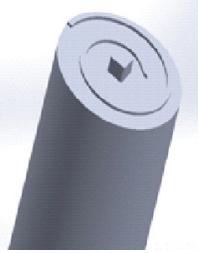

(d)
Fig. 3. Tool Shoulder Profiles having scroll direction (a) CW and (b) ACW with cylindrical pin, and (c) $\mathrm{CW}$ and (d) ACW with square pin.

all FSP runs the tool rotational direction, rotational speed, traverse rate, and tilt were fixed at CW, $900 \mathrm{rpm}, 40 \mathrm{~mm}$ / min, and $2.5^{\circ}$, respectively. The FSPed samples were sectioned and polished using standard metallographic procedure and microscopically analysed. For examination of microstructure extended flick reagent $(15 \mathrm{~mL} \mathrm{HCL}$, $10 \mathrm{~mL} \mathrm{HF}$ and $90 \mathrm{~mL}$ of distilled water) was applied on the samples for $3 \mathrm{~min}$. For macroscopic examination Stereozoom microscope (Focus, Japan) was used and microstructural examination was carried out using OM (QS Metrology, India) and SEM (JEOL JSM 6610).

\section{Results and discussion}

Macroscopic view of cross-section of processed samples is shown in Figure 4a-h. As the samples were FSPed using different tool profile with same set of conventional parameters, different bead geometry profile and presence of various defects such as tunnel and particle agglomeration as shown in Figure 4a-d indicates the significant effect of tool profiles.

For flat shoulder, distribution of particles is better when square pin is used, but at the same time it also leads to agglomeration of $\mathrm{SiC}$ in SZ. Macrographs of samples processed with CW scroll (Fig. 4e-f) exhibit that for tools with scrolled shoulder, much smaller reinforced region is produced as compared to tool with ACW scroll (Fig. $4 \mathrm{~g}-\mathrm{h}$ ). In both the samples with CW scroll (Fig. 5a, b), however, a layer of material from the surface was removed during processing, creating loss of material and reinforcement both as well as very rough top surface. As shoulder diameter was constant, the variation in width of processed 

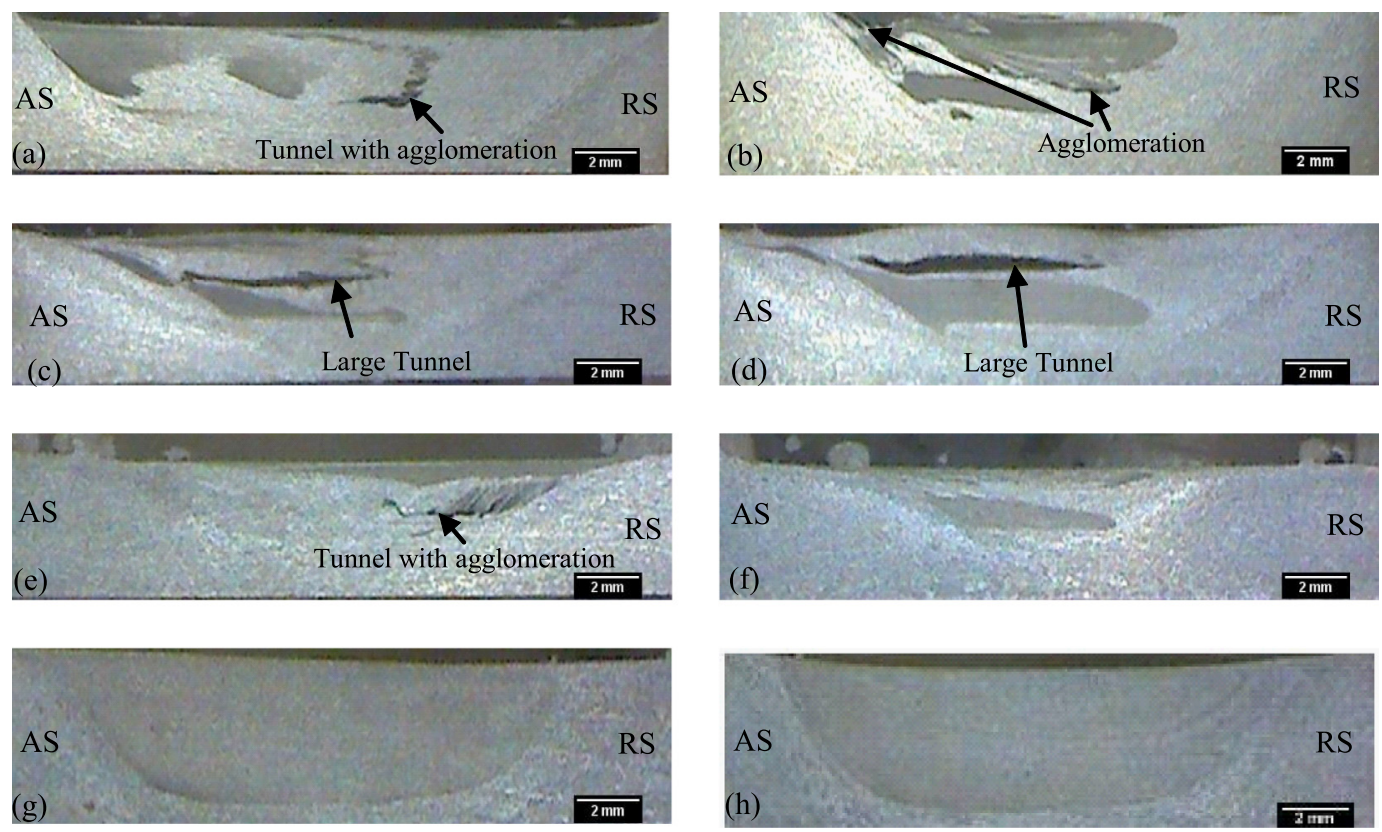

Fig. 4. Macroscopic images of cross-sections of samples processed with (a) Plain cylindrical pin, (b) Square pin, (c) Tapered cylindrical pin, (d) Triangular pin, (e) CW Scrolled shoulder with cylindrical pin, (f) CW scrolled shoulder with square pin, (g) ACW scrolled shoulder with cylindrical pin (h) ACW scrolled shoulder with square pin.

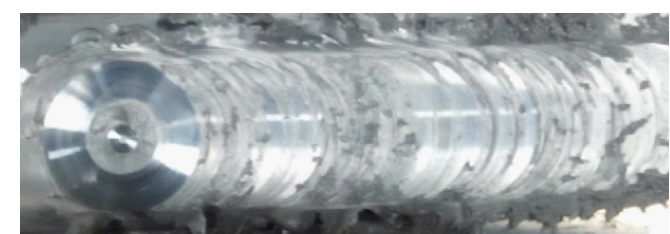

(a)

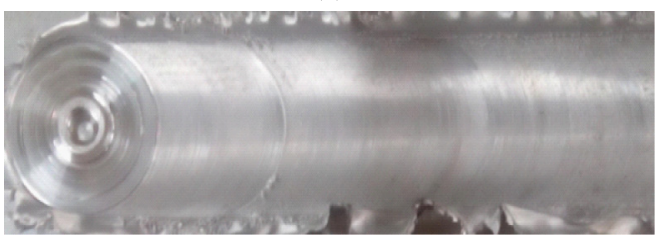

(c)

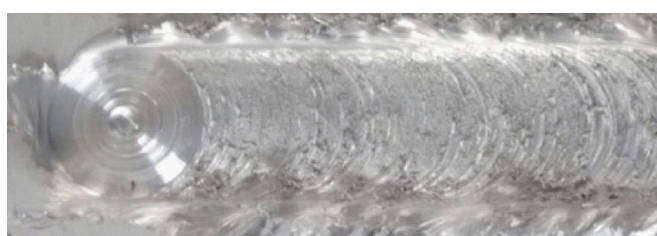

(b)

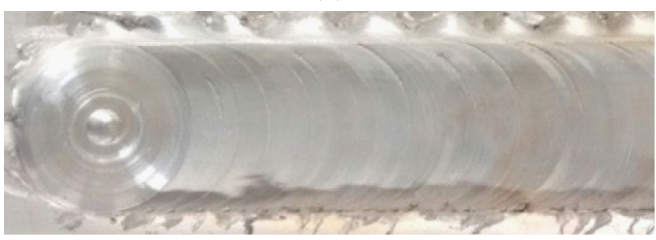

(d)

Fig. 5. Top surface of plates after FSP using CW scroll with (a) cylindrical pin, (b) square pin, and ACW scroll with (c) cylindrical pin, (d) square pin.

zone under the shoulder would not be significant, consequently instead of width, the depth of processed zone and processed area were considered for analysis of effect of tool profile. Other details of the processed region such as size of defect, depth and area of reinforced zone are given in Table 1 which reveal that ACW scrolled shoulder gave a bigger and deeper composite zone.

Optical micrographs of all the samples are presented in Figure $6 \mathrm{a}-\mathrm{f}$. It is evident that ACW scroll with square pin gave bands of mixed and unmixed regions (Fig. 6c-d), while ACW scroll with cylindrical pin did not give any such bands and produced homogeneous distribution of particles in aluminum matrix (Fig. 6e, f). SEM images in Figure 7a indicate a good bonding at the interface between the reinforcement particles and aluminum matrix in the composite zone and, Figure 7b shows uniform distribution of particles in aluminum matrix with average grain size of $4.5 \mu \mathrm{m}$.

As the tool profile affects rate of heat generation, torque and traversing force during FSP as well [13], consequently, it significantly affects the material movement specifically when the FSP conditions favour lower heat. Like several studies a typical investigation by Mahmoud et al. also supports this view and reports that among various pin profiles (plain cylindrical, cylindrical threaded, square and triangular) the tool with square pin profile (at three passes of FSP) more homogeneously distributes $\mathrm{SiC}$ particles in aluminum matrix [7]. Under such conditions the processing forces and resistance of material to flow also increase which leads to defects like tunnel hole [10,11]. Flat faced 
Table 1. Reinforced zone depth, area and tunnel defect dimensions at various tool profiles.

\begin{tabular}{lllcc}
\hline S.No & \multicolumn{1}{c}{ Tool profiles } & $\begin{array}{c}\text { Tunnel } \\
\text { dimension }(\mathrm{mm})\end{array}$ & $\begin{array}{c}\text { Reinforced zone } \\
\text { depth }(\mathrm{mm})\end{array}$ & $\begin{array}{c}\text { Reinforced zone } \\
\text { area }\left(\mathrm{mm}^{2}\right)\end{array}$ \\
\hline 1 & Plain cylindrical pin & 0.4 & 2.04 & 16.27 \\
2 & Tapered cylindrical pin & 1.2 & 2.37 & 6.59 \\
3 & Square pin & - & 2.62 & 22.59 \\
4 & Triangular pin & 2.9 & 2.38 & 11.9 \\
5 & CW scroll with cylindrical pin & 0.3 & 1.82 & 8.07 \\
6 & ACW scroll with cylindrical pin & - & 3.33 & 43.02 \\
7 & CW scroll with Square pin & - & 1.91 & 10.24 \\
8 & ACW scroll with square pin & - & 3.34 & 43.16 \\
\hline
\end{tabular}
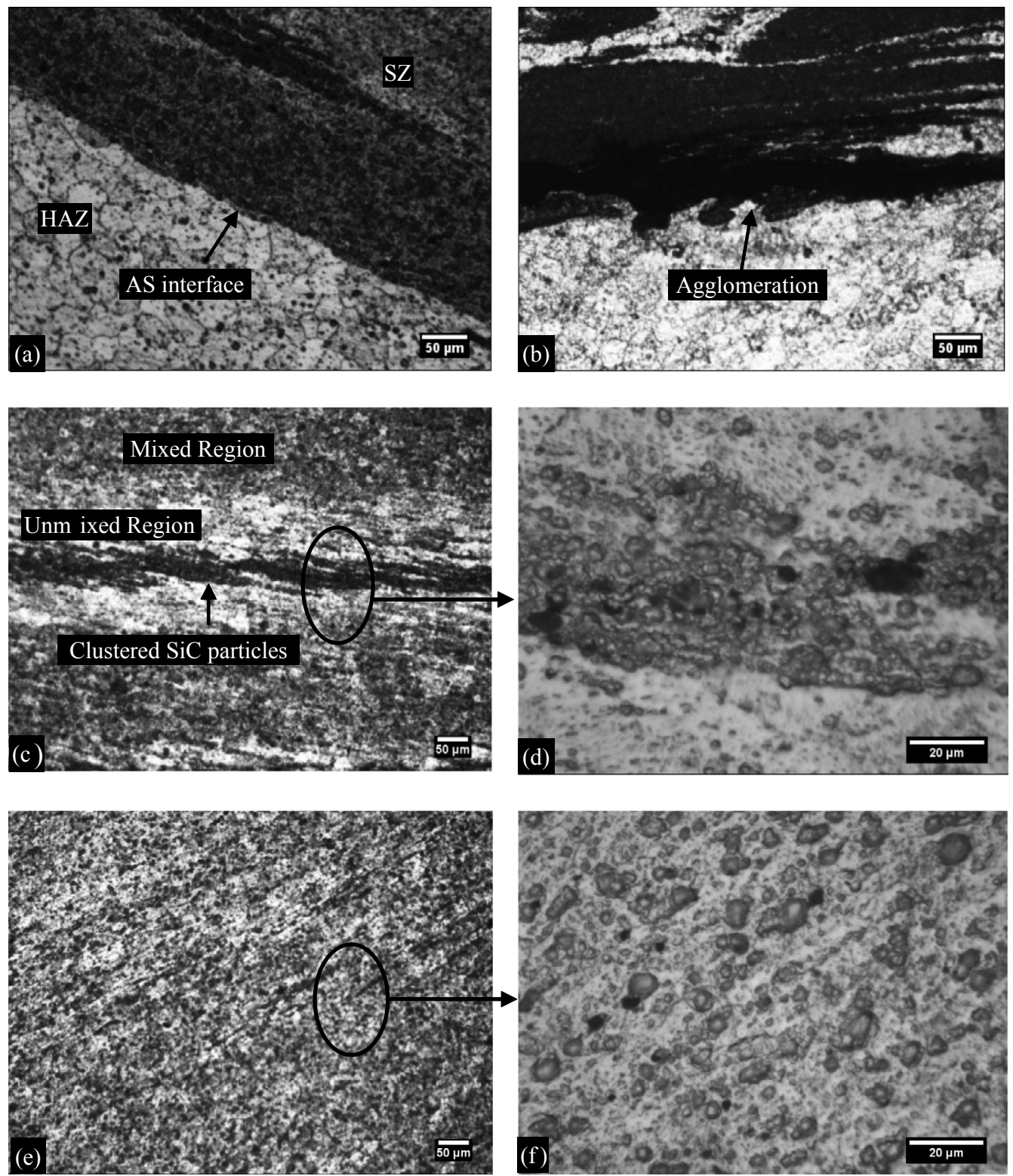

Fig. 6. Optical photomicrographs of sample processed with (a, b) flat shoulder with Square pin, (c, d) ACW Scrolled shoulder with square pin, and (e,f) ACW scrolled shoulder with cylindrical pin. 

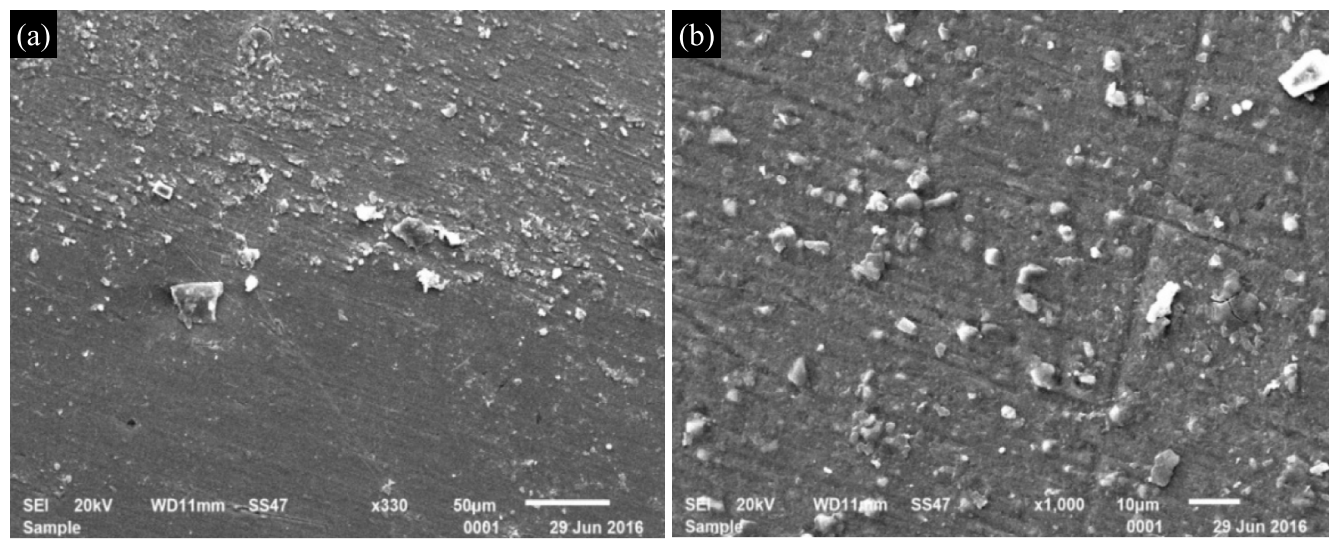

Fig. 7. SEM micrographs of cross-sections of sample processed with ACW scrolled shoulder with cylindrical pin showing (a) Interface of SZ and BM, and (b) SZ.

pin/probe (like in square and triangular pin) generates higher torque and hence effective stirring action of tool $[7,14]$. In the present study samples processed using square pin did not have defects such as tunnel defect as is evident from Figure $4 \mathrm{~b}$ and achieved better particle distribution as compared to other pin profiles. This may be attributed to pulsating stirring action caused by periodic changes in pressure and velocity vector of material by rotating tool (as the faces of pin periodically change orientation with velocity vector) $[7,10,14]$.

Shoulder provides major portion of heat to the surface and subsurface region of workpiece material. Further, shoulder surface with profiles such as scroll, concavity and convexity etc. is an important aspect of tool design [13]. The channel in scrolled shoulder directs plasticized material from periphery towards the axis [2] and viceversa depending on its direction, and hence, may be advantageous over flat shoulder surface.

The combined effect produced by tool shoulder and pin profile is responsible for flow, particle distribution and microstructure of SZ. Shoulder facilitates bulk material transfer while the tool pin is responsible for layer by layer material flow [15]. Typically, three possible combinations of material flow, termed as rigid disc rotational, uniform translational, and ring vortex flow field respectively, are presented in Schneider and Nunes model [16]. The ring vortex flow is driven by pin threads driving metal up on the outside, inward at the shoulder surface, downwards from the pin threads, and outward again from the lower part of the pin. The vortex flow direction changes on reversing the direction of tool rotation $[16,17]$. The linear and rotational direction of tool is equally important as it affects the flow of material [13]. In the study of Chowdhury et al. [17] the CW threaded pin tool in $\mathrm{CW}$ rotational direction exhibited porosity and poor bonding defects near the bottom surface of welded joints due to reversed vortex flow direction as mentioned above [17]. In light of this, it is clear that the orientation of scrolls on shoulder with respect to tool rotational direction plays an important role in material flow. It is observed that the $\mathrm{CW}$ and $\mathrm{ACW}$ scrolls on shoulder result in reversal of material flow. With CW tool rotation, $\mathrm{CW}$ scrolls drive the material to flow outwards that causes loss of material in the form of flash and rough top surface as shown in Figure 4e-f and Figure 5a, b, while ACW scrolls direct the material inwards which results in negligible flash and smooth top surface with bigger and deep reinforced zones as shown in Figures $4 \mathrm{~g}, \mathrm{~h}$ and Figure 5c, d.

In addition the use of cylindrical pin along with ACW scrolls on shoulder creates more homogeneous microstructure among other shoulder and pin profile combinations. This happens due to smooth flow occurring by the use of cylindrical tool profile [18] and better flow path and forging action created by ACW scroll. The improvement in processed zone with ACW scrolled shoulder is attributed to combined effect of shoulder and pin geometry which could effectively and widely distribute particles.

\section{Conclusion}

The present study demonstrated that tool geometry plays very important role apart from of the standard FSP process parameters. FSP tool pin and shoulder profile greatly affect the shape and size of processed zone, particle distribution and microstructure of surface layer of $\mathrm{SiC}$ reinforced composite on AA6063-T6 aluminum alloy. The result obtained can be concluded as follows:

- flat shoulder with cylindrical taper and triangular pin and, clockwise scrolled shoulder with square and cylindrical pin were found ineffective;

- flat shoulder with square pin distributes particles better vis-a-vis other pin profiles when used with flat shoulder;

- bigger and deeper pancake shaped composite zone is achieved by using anti-clockwise scrolled shoulder profiles;

- anti-clockwise scrolled shoulder with cylindrical pin tool geometry found best among others with uniform distribution of particles and no defects.

\section{Nomenclature}

$\begin{array}{ll}\text { ACW } & \text { Anti Clockwise } \\ \text { BM } & \text { Base Metal } \\ \text { CW } & \text { Clockwise }\end{array}$

FSP Friction Stir Processing 


$\begin{array}{ll}\text { FSW } & \text { Friction Stir Welding } \\ \text { HCHCr } & \text { High Carbon High Chromium } \\ \text { HCl } & \text { Hydrochloric Acid } \\ \text { HF } & \text { Hydrofluoric Acid } \\ \text { mm } & \text { Millimetre } \\ \mathrm{ml} & \text { Millilitre } \\ \mu m & \text { Micrometer } \\ \text { OM } & \text { Optical Microscopy } \\ \text { rpm } & \text { Revolutions Per Minute } \\ \text { SEM } & \text { Scanning Electron Microscopy } \\ \text { SiC } & \text { Silicon Carbide } \\ \text { SZ } & \text { Stir Zone } \\ \text { TWI } & \text { The Welding Institute }\end{array}$

Acknowledgements. The authors wish to thank the University Grants Commission (UGC) for its financial assistance (vide sanction order No. F.3-40/2012(SAP-II)) under its SAP (DRS-I) sanctioned to Department of Mechanical Engineering, Jamia Millia Islamia, New Delhi for the project entitled "Friction Stir Welding, Ultrasonically Assisted Machining".

\section{References}

[1] W.M. Thomas, E.D. Nicholas, J.C. Needham, M.G. Murch, P. Templesmith, C.J. Dawes, International Patent Application PCT/GB92/02203 and GB Patent Application No.9125978.8, 1991, UK Patent Office, London

[2] R.S. Mishra, M.W. Mahoney, Friction stir processing, in: R. S. Mishra, M.W. Mahoney (Eds.), Friction Stir Welding and Processing, ASM International, 2007, pp. 309-350

[3] N.Z. Khan, A.N. Siddiquee, Z.A. Khan, S.K. Shihab, Investigations on tunneling and kissing bond defects in FSW joints for dissimilar aluminum alloys, J. Alloy. Compd. 648 (2015) 360-367

[4] A.N. Siddiquee, S. Pandey, N.Z. Khan, Friction stir welding of austenitic stainless steel: a study on microstructure and effect of parameters on tensile strength, Mater. Today Proc. 2 (2015) 1388-1397

[5] A. Heydarian, K.N. Dehghani, T. Slamkish, Optimizing powder distribution in production of surface nano-composite via friction stir processing, Metall. Mater. Trans. B 45B (2014) 821-826
[6] R. Hashemi, G. Hussain, Wear performance of $\mathrm{Al} / \mathrm{TiN}$ dispersion strengthened surface composite produced through friction stir process: a comparison of tool geometries and number of passes, Wear 324 (2015) 45-54

[7] E.R.I. Mahmoud, M. Takahashi, T. Shibayanagi, K. Ikeuchi, Effect of friction stir processing tool probe on fabrication of $\mathrm{SiC}$ particle reinforced composite on aluminium surface, Sci. Technol. Weld. Join. 14 (2009) 413-425

[8] A. Dolatkhah, P. Golbabaei, M.K.B. Givi, F. Molaiekiya, Effects of process parameters on microstructural and mechanical properties of $\mathrm{Al} 5052$ / $\mathrm{SiC}$ metal matrix composite fabricated via friction stir processing, Mater. Des. 37 (2012) 458-464

[9] T.G. Santos, N. Lopes, M. Machado, P. Vilaca, R.M. Miranda, Surface reinforcement of AA5083-H111 by friction stir processing assisted by electrical current, J. Mater. Process. Technol. 216 (2015) 375-380

[10] K. Elangovan, V. Balasubramanian, Influences of pin profile and rotational speed of the tool on the formation of friction stir processing zone in AA2219 aluminium alloy, Mater. Sci. Eng. A 459 (2007) 7-18

[11] K. Elangovan, V. Balasubramanian, Influences of tool pin profile and tool shoulder diameter on the formation of friction stir processing zone in AA6061 aluminium alloy, Mater. Des. 29 (2008) 362-373

[12] M. Dixit, J.W. Newkirt, R.S. Mishra, Properties of friction stir-processed Al 1100-NiTi composite, Scr. Mater. 56 (2007) $541-544$

[13] R. Rai, A. De, H.K.D.H. Bhadeshia, T. DebRoy, Review: friction stir welding tools, Sci. Technol. Weld. Join. 16 (2011) 325-342

[14] P.A. Colegrove, H.R. Shercliff, Two-dimensional CFD modelling of flow round profiled FSW tooling, Sci. Technol. Weld. Join. 9 (2004) 483-492

[15] K. Kumar, S.V. Kailas, The role of friction stir welding tool on material flow and weld formation, Mater. Sci. Eng. A 485 (2008) 367-374

[16] J.A. Schneider, A.C. Nunes Jr, Characterization of plastic flow and resulting microstructures in a friction stir weld, Metall. Mater. Trans. B 35 B (2004) 777-783

[17] S.M. Chowdhury, D.L. Chen, S.D. Bhole, X. Cao, Effect of pin tool thread orientation on fatigue strength of friction stir welded AZ31B-H24 Mg butt joints, Procedia Eng. 2 (2010) $825-833$

[18] T.U. Seidel, A.P. Reynolds, Two-dimensional friction stir welding process model based on fluid mechanics, Sci. Technol. Weld. Join. 8 (2003) 175-183 